\title{
Research on Segmentation Technology of Fuzzy Image with Adhesion Pixels
}

\author{
Zhang Yun-Peng*
}

Department of Computer, Dongying Vocational College, No.129, Fu Qian Road, Dongying City, Shandong Province, 257091, China

\begin{abstract}
In a blurred image, the difference of gray level between adhesion pixels and the pixels of background image is small, so it is difficult to accurately segment. Traditional methods for segmenting fuzzy image with adhesion pixels assume that the image is large, and then the computational complexity dramatically expands, which reduces the accuracy of segmentation. In order to solve this problem, this article puts forward a method of fuzzy image segmentation with adhesion pixels based on similarity probability. Using the characteristic values of the Hessian matrix to detect the adhesion pixels, the probability whether the pixel in the image belongs to adhesion pixel can obtain. Applying the method of Gaussian gradient convolution processing, the edge enhanced image of adhesion pixel area can be achieved. Taking advantage of circular probe, the accurate adhesion points can be obtained, so as to complete accurate segmentation of adhesion pixels in the blurred image. The experimental results show that the algorithm presented in this paper for segmenting fuzzy image with adhesion pixels can greatly improve the segmentation accuracy.
\end{abstract}

Keywords: Adhesion pixels, fuzzy image, image segmentation, probability similarity.

\section{INTRODUCTION}

With the rapid development of image processing technology, image processing technology has been playing a more and more important role in aerospace, medical, agriculture, meteorology, military, and many other areas [1]. In the procedure of fuzzy image processing, how to effectively segment adhesion pixels from the blurred images has become a core issue needed to study in the field of image processing [2], which is focused on by many experts. The segmenting effect of adhesion pixels from a fuzzy image has great influence for image feature extraction and target recognition [3], the related research institutes have invested a lot of resources on segmentation technology research of adhesion pixels from fuzzy images [4]. Currently, the main methods for segmenting adhesive pixels from fuzzy images include 2D maximum entropy algorithm with two adhesion pixels [5], segmenting method based on genetic algorithm [6] and segmenting method based on Normalized Cut algorithm [7]. In which, one of the most commonly used is based on the Normalized Cut algorithm of adhesion pixels fuzzy image segmentation method [8]. Due to the segmentation method of fuzzy image with adhesion pixels plays an irreplaceable role in many area, therefore, it has become the important research topic of many experts and has wide development space [9].

In the procedure of segmenting fuzzy image with adhesion pixels using traditional algorithms, there is a problem.
Supposing that the image you need to split is too large, the computational complexity of the algorithm increases sharply, leading to accuracy reduction. Therefore, how to quickly and accurately segment fuzzy images with adhesion pixels has become a difficult problem in the field of image processing [10].

In order to avoid the disadvantages of the traditional algorithms, this paper puts forward a method of fuzzy image segmentation with adhesion pixels based on similarity probability. Using the characteristic values of the Hessian matrix to detect the adhesion pixels, the probability whether the pixel in the image belongs to adhesion pixel can obtain. Applying the method of Gaussian gradient convolution processing, the edge enhanced image of adhesion pixel area can be achieved. Taking advantage of circular probe, the accurate adhesion points can be obtained, so as to complete accurate segmentation of adhesion pixels in the blurred image. The experimental results show that the algorithm presented in this paper for segmenting fuzzy image with adhesion pixels can avoid the disadvantages of traditional algorithms, and greatly improve the accuracy of segmentation.

\section{PRINCIPLE OF FUZZY IMAGE SEGMENTATION WITH ADHESION PIXELS}

Normalized Cut can effectively segment the fuzzy image with adhesion pixels. The core thought of this image segmentation method is to set up a non-vector image with weight value of a blurred image needs to be segmented. Each pixel in the blurred image with adhesion pixels needs to be segmented is a node in the non-vector image. Searching for a standardized minimum segmenting region in the non-vector image is dividing all nodes in the region. This method of 
image segmentation does not need initialization processing, and has the following three characteristics at the same time: (1) The image segmentation method can transform the problem of the blurred image with adhesive pixels to classification of non-vector images nodes. (2) The image segmentation method has global standards. (3) The image segmentation method can maximized display the similarity between different regions and the similarity of the same region at the same time. The method of segmenting blur images with adhesion pixels through Normalized Cut is described below.

Setting an pixels collection in any region of the fuzzy image with adhesion pixels can be described as a non-vector image with weight value, for an image with weight value $M(P, Q)$, hypothesis it can be divided into two relatively independent regions $C$ and $D, C \cup D=P$. Moving the connected edge of $A$ and $B$ can divided the image into two regions, and then a partition function could be described between through the following formula:

$\operatorname{cut}(C, D)=\sum_{i \in C, j \in \Delta} u(h, k)$

In the formula above, $u(h, k)$ is a weight function, which can be used to describe the degree of similarity between two nodes.

The image segmentation method of Normalized Cut can compare the cut value between $C$ and $D$ with the sum of the weights of $C$ to all the nodes in the image and the sum of the weights of $D$ to all the nodes in the image, and calculates the accumulative sum of the two ratio. The formula is as follows:

$$
\operatorname{Ncut}(C, D)=\frac{\operatorname{cut}(C, D)}{\operatorname{assoc}(C, P)}+\frac{\operatorname{cut}(C, D)}{\operatorname{assoc}(d, P)}
$$

In the formula above,

$$
\begin{aligned}
& \operatorname{assoc}(C, P)=\sum_{z \in \mathrm{C}, l \in p} u(z, l) \\
& \operatorname{assoc}(D, P)=\sum_{z \in \mathrm{D}, l \in p} u(z, l)
\end{aligned}
$$

For the fuzzy image $M$, searching for the optimal segmentation method which can make Ncut between $C$ and $D$ minimum is needed.

Setting $\mathrm{n}=|\mathrm{P}|, \mathrm{y}$ is used to describe a vector with $\mathrm{n}$ dimensions. $y=1$ is used to describe that the node $h$ is a pixel in fuzzy image $C$ region, and $\mathrm{y}=-1$ is used to describe that the node $\mathrm{h}$ is a pixel in fuzzy image $D$ region. $\mathrm{U}$ is a $\mathrm{n} \times \mathrm{n} \quad$ symmetric matrix, and $\mathrm{U}(\mathrm{h}, \mathrm{k})=\mathrm{u}(\mathrm{h}, \mathrm{k})$, $a_{h}=\sum_{k} u(h, k), A$ is a diagonal matrix, and $A(h, k)=a_{h}$, $r=\frac{\sum_{y_{h}>0} a_{h}}{\sum_{h} a_{h}}, v$ is a $n \times 1$ vector with all pixels are 1, then $\operatorname{Ncut}(C, D)$ can be converted the following functions:

$\min _{y} \operatorname{Ncut}(y)=\min _{x} \frac{x^{s}(A-E) x}{x^{S} A x}$

According to the formula above, we can learn that the form of the function is the quotient of Rayleigh. Assuming that the value ranges of $x$ has no requirements, and then the solving functions of the minimum of Ncut can be translated into the following general matrix characteristic function:

$(A-E) x=\varepsilon A x$

In the process of solving the minimum value, $x$ will be used as an indicator vector, and using the image segmentation method of Normalized Cut can make the characteristic vector corresponding to the second minimum value be the solution to the problem of fuzzy image segmentation with the adhesion pixel. Selecting a split value in the vector $x$, make the nodes corresponding to the larger than the numerical part in the region $C$ of the image, the rest of the nodes in the region $D$ of the image. Using the method above can divide nodes in the blurred image $M$ with adhesion pixels into two parts. Usually, 0 is the dividing point, if the dividing part need to be divided a second time, then the method above can be recursive calls, finally achieving effectively segmenting the fuzzy image with adhesion pixels.

\section{IMAGE SEGMENTATION METHOD BASED ON SIMILARITY PROBABILITY}

Using traditional algorithm for fuzzy image segmentation with adhesion pixels, as a result of the segmentation process is to clustering points in the blurred image, therefore, assuming that the data volume in the blurred image increases, computational complexity of segmentation algorithm will increase sharply, leading to accuracy reduction. For this, the article puts forward an image segmentation method with adhesion pixels based on similarity model.

\subsection{Detection of Adhesion Pixels}

In the procedure of segmenting adhesion pixels from fuzzy images, the computation of similarity of adhesion pixels in the blurred image is needed. Setting the Hessian matrix $H=\left[I_{x x}, I_{x y}, I_{y x}, I_{y y}\right]$ is the collection of the second order partial derivative of a specific point $(x, y)$ in a blurred image $I$. Whether a specific point $x_{0}$ belongs to the adhesion pixel can be judged from the characteristic value of the Hessian 
matrix. Assuming that the adhesion pixels and background pixels are respectively corresponding to two different regions, and the characteristic value of the pixel $(x, y)$ conform to $\left|\lambda_{1}\right|>\left|\lambda_{2}\right|$, the similarity function of adhesion pixel can be described by the following formula:

$$
Z\left(\lambda_{1}, \lambda_{2}\right)=\left\{\begin{array}{c}
0 ;\left(\text { if } \lambda_{2}>0\right) \\
\exp \left(-\frac{R_{B}^{2}}{2 \beta^{2}}\right)\left[1-\exp \left(-\frac{S^{2}}{2 c^{2}}\right)\right]
\end{array}\right.
$$

in which, $R_{B}=\lambda_{1} / \lambda_{2} ; S=\sqrt{\lambda_{1}^{2}+\lambda_{2}^{2}}$

\subsection{Maximum Similarity Estimation of Adhesion Pixels}

Using the calculation of maximum likelihood probability can obtain the estimation results of maximum similarity of adhesion pixels. Assuming that $Z^{\prime}=\left\{(X, Y) \mid \mathrm{Z}(\mathrm{x}, \mathrm{y})>\mathrm{T}_{\mathrm{z}}\right\}$ is used to describe the adhesion pixel area, then we can obtain clear adhesive pixels after refining processing of $Z^{\prime}$. Conditions based on the characteristics of the pixel, distance and direction consistency can be calculated by the similarity condition probability estimation. The following formula can describe the corresponding relationship function of sampling event of adhesion pixels and the probability.

$$
\begin{aligned}
& Z^{\prime}\left(\mathrm{X}_{\mathrm{t}+1}^{\mathrm{i}}\right) ; \sigma\left(\mathrm{X}_{\mathrm{t}+1}^{\mathrm{i}}, \mathrm{L}_{\mathrm{c}}\right) ; \\
& \angle\left(\mathrm{V}_{\mathrm{T}+1}^{\mathrm{I}}, \mathrm{V}_{\mathrm{t}}\right) \mid \mathrm{X}_{\mathrm{t}} \rightarrow \mathrm{P}\left(\mathrm{X}_{\mathrm{t}+1} \mid \mathrm{X}_{\mathrm{t}}\right)
\end{aligned}
$$

Thus the probability calculation function of formula (7) on its left can be obtained as follows:

$$
\begin{aligned}
& \mathrm{D}^{\prime}\left(\mathrm{X}_{\mathrm{t}+1}^{\mathrm{i}}\right)=\frac{1}{\mathrm{~T}_{1}} \exp \left(\frac{\left|\delta\left(\mathrm{X}_{\mathrm{t}+1}^{\mathrm{i}}, \mathrm{L}_{\mathrm{c}}\right)\right|^{2}}{-2 \sigma_{\mathrm{D}}^{2}, \mathrm{t}}\right) \\
& \varphi_{\mathrm{Z}}\left(\mathrm{X}_{\mathrm{t}+1}^{\mathrm{i}}\right)=\frac{1}{\mathrm{~T}_{2}} \exp \left(\frac{\mid \mathrm{Z}_{\max }-\mathrm{Z}\left(\mathrm{X}_{\mathrm{t}+1}^{\mathrm{i}}\right)^{2}}{-2 \sigma_{\mathrm{Z}}^{2}, \mathrm{t}}\right) \\
& \theta\left(\mathrm{X}_{\mathrm{t}+1}^{\mathrm{i}}\right)=\frac{1}{\mathrm{~T}_{3}} \exp \left(\frac{\left|\angle\left(\mathrm{V}_{\mathrm{t}+1}^{\mathrm{i}}, \mathrm{V}_{\mathrm{t}}\right)\right|^{2}}{-\sigma_{\theta}^{2}}\right)
\end{aligned}
$$

According to the formula above, we know that the maximum similarity probabilities of adhesion pixels in blurred images can be calculated by the following formula:

$$
\mathrm{X}_{\mathrm{t}+1}=\max _{\mathrm{i}}\left[\mathrm{D}\left(\mathrm{X}_{\mathrm{t}+1}^{\mathrm{i}}\right) \cdot \varphi_{\mathrm{Z}}\left(\mathrm{X}_{\mathrm{t}+1}^{\mathrm{i}}\right) \cdot \theta\left(\mathrm{X}_{\mathrm{t}+1}^{\mathrm{i}}\right)\right]
$$

The result of formula (11) $\mathrm{X}_{\mathrm{t}+1}$ is the pixel on the initial adhesion pixels region $\mathrm{L}_{\mathrm{c}}$, using the Gaussian gradient convolution processing method, the edge enhanced image $\mathrm{g}(\mathrm{x}, \mathrm{y})$ of adhesion pixel region can be obtained, and form- ing two Gaussian gradient curves in the nodes of the blurred image.

Using the following formula to calculate the center of adhesion pixels:

$$
g\left(P_{k}=\frac{1}{2} \max _{k=1: M}\left[\sum_{i=k}^{k-w} g\left(P_{i}\right)+\sum_{j=k}^{k+w} g\left(P_{i}\right)\right]\right.
$$

In the formula above, $\mathrm{w} \leq \min (\mathrm{M}-\mathrm{k}, \mathrm{k}-1)$, in which, $\mathrm{M} \approx 2 \max _{\mathrm{n}}\left(\sigma_{\mathrm{n}}\right) . \mathrm{P}_{\mathrm{k}}(\mathrm{x}, \mathrm{y})$ is the adhesion pixel.

\subsection{Segmenting Adhesion Pixels}

Circular probe method is used to scan the initial fuzzy image $L_{c}(x, y)$, and $M$ adhesion points can be obtained, judging the adhesion points through the method of probability pattern recognition, and accurately segmenting the pixels. The specific method is as follows:

First of all, we need to get four characteristics of the $m_{\text {th }}$ adhesion point: (1) the characteristic data of adhesion pixel $Z(m)$. (2) the vector $V_{O, m}$ from detector center $O_{t}$ to the adhesion pixel m. (3) and (4) are used to describe the amplitude $\left|\psi_{\mathrm{m}}\right|$ of response of the Gabor filter $\left|\psi_{\mathrm{m}}\right| \mathrm{e}^{\mathrm{i}=\theta_{\mathrm{m}, t}}$ and its phase angle $\theta_{\mathrm{m}, \mathrm{t}}$ at the direction of $\mathrm{V}_{\mathrm{m}}$. Using the four kinds of characteristic data of adhesion pixel above to structure similarity subsets on the set of $U_{m}=\{m \mid m=1,2, \ldots, M\}$, respectively, then apply the following logarithmic function to describe:

$$
\left\{\begin{array}{c}
\rho_{\mathrm{Z}}(\mathrm{m})=\mathrm{Z}_{\mathrm{m}}^{2} / \mathrm{Z}_{\max }^{2} \\
\rho_{\mathrm{V}}(\mathrm{m})=\mid \cos \angle\left(\mathrm{V}_{\mathrm{o}, \mathrm{m}}-\left.\mathrm{V}_{\mathrm{m}}\right|^{2}\right. \\
\rho_{\psi}(\mathrm{m})=\psi_{\mathrm{m}}^{2} / \psi_{\text {max }}^{2} \\
\rho_{\theta}(\mathrm{m})=\mid \cos \angle\left(\mathrm{V}_{\mathrm{o}, \mathrm{m}}-\left.\theta_{\mathrm{m}, \mathrm{t}}\right|^{2}\right.
\end{array}\right.
$$

The similarity subset $\mathrm{F}$ of collection $\mathrm{U}_{\mathrm{m}}$ can be used to describe the similarity degree whether the pixel $\mathrm{m}$ belongs to adhesion pixel, then using the following formula to describe the relations between $\mathrm{F}$ and the four sets above:

$$
\mathrm{F}=\mathrm{Z} \cap \mathrm{V} \cap \psi \cap \theta
$$

Degree of certainty of pixel $\mathrm{m}$ being adhesion pixel mainly depends on the similarity of $\mathrm{F}$. Therefore, the adhesion pixels based on a variety of similarity can be effectively segmented by the following similarity function:

$\rho_{\mathrm{F}}(\mathrm{m})=\min \left\{\rho_{\mathrm{Z}}(\mathrm{m}), \rho_{\mathrm{V}}(\mathrm{m}), \rho_{\psi}(\mathrm{m}), \rho_{\theta}(\mathrm{m})\right\}$

When $\rho_{\mathrm{F}}(\mathrm{m})>\mathrm{T}_{\mathrm{a}}$, then the segmentation result of adhesion pixel points in the image will be affected. 


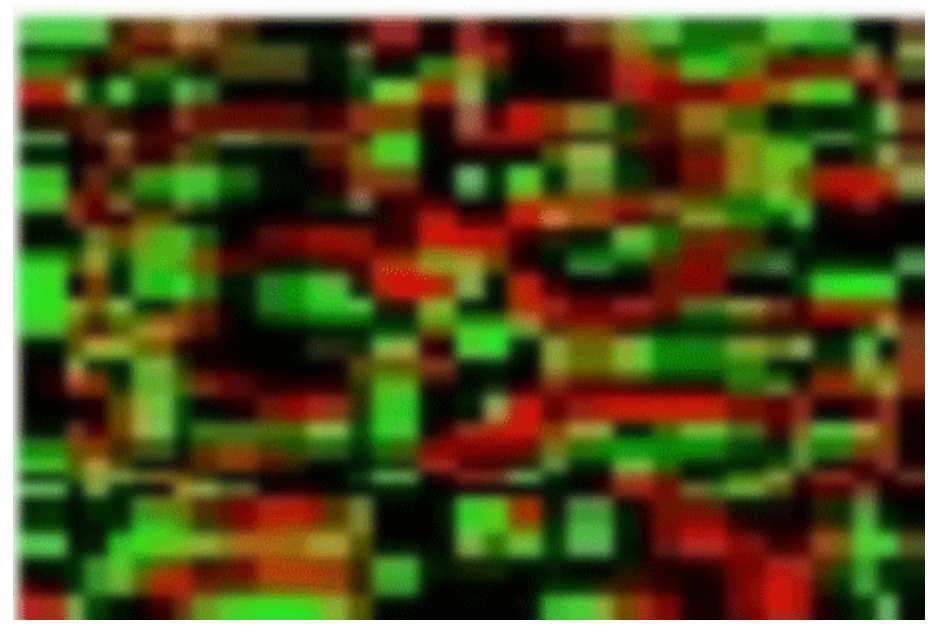

Fig. (1). Original blurred image.

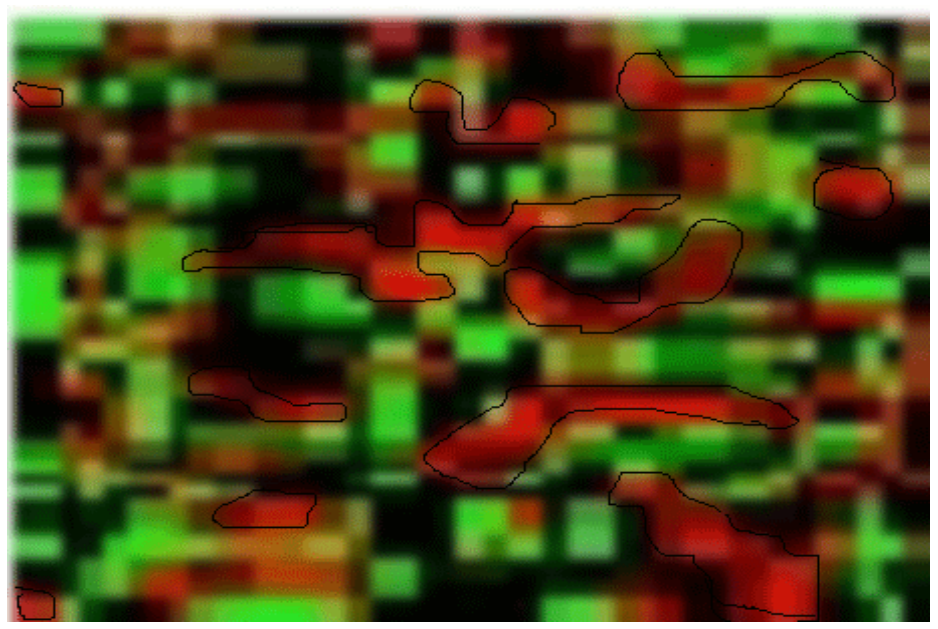

Fig. (2). Result of segmentation by traditional algorithm.

\section{ANALYSIS OF EXPERIMENTAL RESULTS}

In order to verify the validity of the algorithm in this paper, an experiment is needed. Using matlab7.1 simulation software to build experimental environment, the segmentation experiments of adhesion pixels in fuzzy image are carried out. Apply different algorithms to segment adhesion pixels in fuzzy image, respectively.

In the process of experiment, an original blurred image is needed for adhesion pixel segmentation, which is described as Fig. (1).

Result of the experiment using traditional algorithm for adhesion pixel segmentation in the blurred image can be described in the following Fig. (2).

Result of the experiment using algorithm presented in this paper for adhesion pixel segmentation in the blurred image can be described in the following Fig. (3).

According to the experiment results above, we could learn that the adhesion pixels in blurred image are mixed together. It is difficult to accurately segment through traditional algorithm, but accurate segmentation result is obtained by the algorithm presented in this paper.

Experiment has been done for 10 times, with the degree of fuzzy increases in the blurred image, using different algorithms to segment adhesion pixels of the fuzzy image, the experiment result obtained can be described in Table $\mathbf{1}$ below:

According to the experimental results in Table 1, we can learn that the algorithm of adhesion pixels segmentation in fuzzy image presented in this paper can greatly improve the accuracy of segmentation, fully shows the superiority of the algorithm in this paper.

\section{CONCLUSION}

In view of the traditional algorithm cannot avoid computational cost increasing dramatically due to the large blurred image in the process of segmenting adhesion pixels in fuzzy 


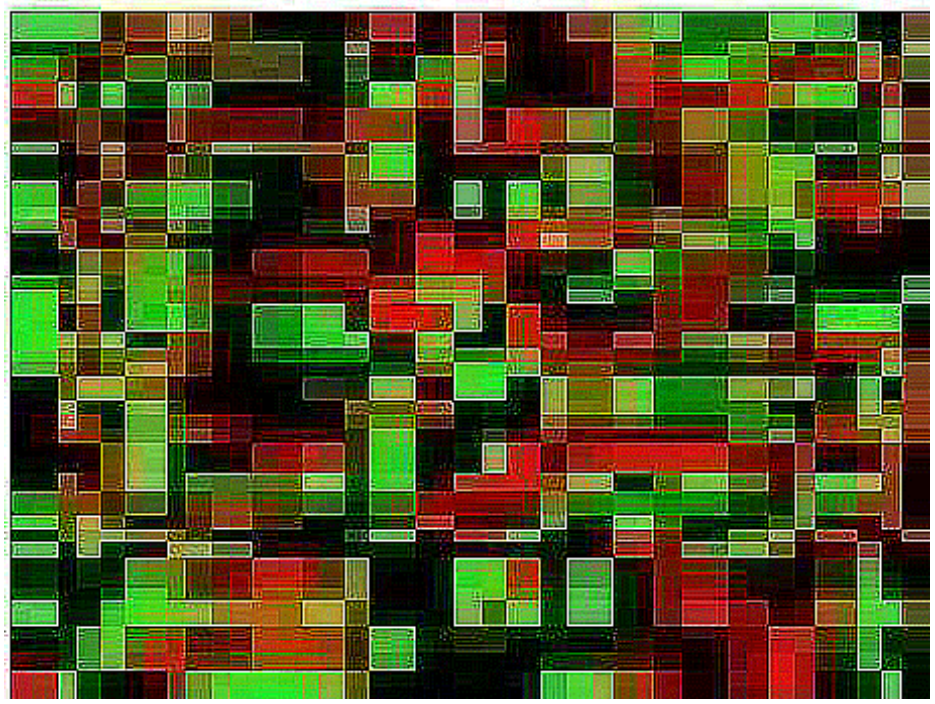

Fig. (3). Result of segmentation by proposed algorithm in this paper.

Table 1. Results of different algorithms.

\begin{tabular}{|c|c|c|c|}
\hline \multirow{2}{*}{ Times of Experiment } & \multirow{2}{*}{ Coefficients of Fuzzy } & \multicolumn{2}{|c|}{ Accuracy of Segmentation (\%) } \\
\cline { 2 - 4 } & & Traditional Algorithm & 97 \\
\hline \hline 1 & 0.02 & 98 & 98 \\
\hline 2 & 0.11 & 75 & 96 \\
\hline 3 & 0.23 & 64 & 96 \\
\hline 4 & 0.34 & 57 & 96 \\
\hline 5 & 0.46 & 49 & 93 \\
\hline 6 & 0.52 & 34 & 96 \\
\hline 7 & 0.64 & 27 & 91 \\
\hline 10 & 0.76 & 18 & 99 \\
\hline
\end{tabular}

image, which leads to the defect reduction of segmentation accuracy. For this, the article puts forward a method of segmenting adhesion pixels in fuzzy image based on similarity probability. Using the characteristic values of the Hessian matrix to detect the adhesion pixels, the probability whether the pixel in the image belongs to adhesion pixel can obtain. Applying the method of Gaussian gradient convolution processing, the edge enhanced image of adhesion pixel area can be achieved. Taking advantage of circular probe, the accurate adhesion points can be obtained, so as to complete accurate segmentation of adhesion pixels in the blurred image. The experimental results show that the algorithm presented in this paper for segmenting fuzzy image with adhesion pixels can greatly improve the segmentation accuracy and satisfactory results have been achieved.

\section{CONFLICT OF INTEREST}

The author confirms that this article content has no conflict of interest.

\section{ACKNOWLEDGEMENTS}

Declared none.

\section{REFERENCES}

[1] Z. Li, and D. Xiao, "Improved image segmentation algorithm of fast fuzzy clustering," Computer Simulation, vol. 26, no. 8, pp. 212-215, 2009

[2] J. Liao, S. Guo, and Z. Huang, "Method of maximum entropy image segmentation based on Mean Shift clustering," Computer Simulation, vol. 26, no. 9 , pp.187-222, 2009. 
[3] Wang, and L. Shen, "Research review of image segmentation," Testing Technology, vol. 12, no. 5, pp.1-6, 2000.

[4] J. Gong, L. Li, and H. Chen, "Image segmentation method based on two-dimensional gray histogram Fisher linear integral," Journal of Pattern Recognition and Artificial Intelligence, vol. 10, pp.1-6, 1997.

[5] Guo, and H. Zuo, "Two dimensional optimal genetic algorithm of image segmentation," Journal of Computer-Aided Design and Graphics, vol. 14, no. 6, pp. 530-536, 2002.

[6] J. Liu, "Image fuzzy clustering segmentation method based on twodimensional histogram," Journal of Electronics, vol. 20, no. 9, pp. 40-46, 1992.
[7] Z. Chen, and W. Fang, "Active contour model and image segmentation algorithm based on distributed matching," Journal of JiLin University (Engineering Science), vol. 38, no. 6, pp. 1441-1446, 2008.

[8] R. Li, and Y. Guo, "Active contour model based on particle swarm optimization algorithm," Journal of Computer Applications, vol. 28 , no. 10 , pp. 2622-2627, 2008

[9] M. N. Do, and V. M. Contourlets, "A directional multi-resolution image representation," In: Proceedings of IEEE International Conference on Image Processing, Rochester: NY, 2002, pp. 357-360.

[10] A.L. Cunha, J. Zhou, and M. N. Do, "The nonsubsampled contourlet transform Theory, design, and applications," IEEE Transactions on Image Processing, vol. 15, no. 10, pp. 3089-3101, 2006.

Received: September 16, 2014

(C) Zhang Yun-Peng; Licensee Bentham Open.

This is an open access article licensed under the terms of the Creative Commons Attribution Non-Commercial License (http://creativecommons.org/licenses/by-nc/3.0/) which permits unrestricted, non-commercial use, distribution and reproduction in any medium, provided the work is properly cited. 